\title{
Generation of internal solitary waves in a pycnocline by an internal wave beam: a numerical study
}

\author{
N. GRISOUARD ${ }^{1} \dagger, C$. STAQUET $T^{1}$ AND T. GERKEMA \\ ${ }^{1}$ Laboratoire des Écoulements Géophysiques et Industriels, UJF/CNRS/G-INP, BP 53, \\ 38041 Grenoble CEDEX 9, France \\ ${ }^{2}$ Royal Netherlands Institute for Sea Research, PO Box 59, 1790 AB Texel, The Netherlands \\ (Received 13 January 2010; revised 20 January 2011; accepted 2 February 2011; \\ first published online 1 April 2011)
}

Oceanic observations from western Europe and the south-western Indian ocean have provided evidence of the generation of internal solitary waves due to an internal tidal beam impinging on the pycnocline from below - a process referred to as 'local generation' (as opposed to the more direct generation over topography). Here we present the first direct numerical simulations of such a generation process with a fully nonlinear non-hydrostatic model for an idealised configuration. We show that, depending on the parameters, different modes can be excited and we provide examples of internal solitary waves as first, second and third modes, trapped in the pycnocline. A criterion for the selection of a particular mode is put forward, in terms of phase speeds. In addition, another simpler geometrical criterion is presented to explain the selection of modes in a more intuitive way. Finally, results are discussed and compared with the configuration of the Bay of Biscay.

Key words: internal waves, ocean processes, solitary waves

\section{Introduction}

Internal solitary waves (ISWs) in the oceans and shelf seas started to be observed in the 1960s; this coincided with mathematical developments on the Korteweg-de Vries $(\mathrm{KdV})$ equation and soliton theory. The KdV equation has since served as the primary theoretical tool for interpreting observations of ISWs in the ocean (Ostrovsky \& Stepanyants 1989). More recently, important developments have occurred on two fronts. First, the advance in measurement techniques such as moored acoustic Doppler current profilers, or airborne and satellite remote sensing, allowing not just detailed but also more synoptic observations to be made. Second, the advance in theoretical and numerical modelling, allowing one to go beyond the weakly nonlinear KdV theory and to study ISWs of large amplitude. These developments, however, have not altered the view of the basic mechanism behind the generation of the majority of ISWs, which starts with (tidal) flow over topography generating internal waves, their subsequent evolution and steepening and finally splitting up into ISWs. In this view, propagation is horizontal, as in unimodal internal or interfacial waves. For a review on the study of ISWs, see Helfrich \& Melville (2006). 
An altogether different mechanism was proposed by New \& Pingree (1990) to explain observations of ISWs in the central Bay of Biscay. They argued that these ISWs are not directly related to topography (the continental slope), but are generated by an internal tidal beam hitting the seasonal thermocline from below. The beam originates from the shelf-break, with downward propagation of energy into the abyssal ocean, which turns into upward propagation when the beam reflects from the bottom. On its upward path, it finally encounters the seasonal thermocline, perturbs it locally and may, under the right circumstances, lead to ISWs in the thermocline. New \& Pingree (1990) coined the term 'local generation' of ISWs to refer to this process. Measurements of the beam location were consistent with ray paths computation based upon the local stratification and the bathymetry of the Bay of Biscay (Pingree \& New 1991). Further evidence of local generation of ISWs was presented by New \& Pingree (1992). This set of three papers therefore convincingly proved the existence of a local generation mechanism of ISWs. Since the beginning of the 2000s, a new remote sensing technique - namely synthetic aperture radar or SAR (New \& da Silva 2002; Azevedo, da Silva \& New 2006) - has confirmed the occurrence of this mechanism in spring and summer, when the seasonal thermocline is present. SAR imagery recently also showed the occurrence of local generation off south-west Portugal (da Silva, New \& Azevedo 2007) and in the Mozambique channel (da Silva, New \& Magalhaes 2009). This mechanism might also explain observations near the Mascarene Ridge by Konyaev, Sabinin \& Serebryany (1995), which, interestingly, shows that the observed ISWs are partly mode-1 waves, i.e. the whole water column in the thermocline moves in phase, and partly mode- 2 waves, in which case the structure of vertical displacements has a node in the middle of the thermocline, while positions above and below this point move in opposite directions. In the remainder of this paper, we shall rather use the term 'pycnocline' in place of thermocline for more generality. Indeed, a temperature jump results in a density jump, as also does a jump in salinity.

Few attempts have so far been made to explain quantitatively the underlying physical mechanism. This mechanism should follow certain rules, requiring specific conditions to be met, as suggested by the sparsity of the observations. We briefly discuss previous work that dealt with this problem.

The effect on a pycnocline of an incident plane internal gravity wave was studied experimentally and theoretically by Delisi \& Orlanski (1975) for the first time, the wave field achievable in their experiments was actually closer to a wave beam. In the theoretical model they developed, the vertical density profile consists of a density jump across an interface (the pycnocline), with a stably stratified layer of constant $N$ above, and a non-stratified layer (i.e. $N=0$ ) below. Here, $N$ is the buoyancy or Brunt-Väisälä frequency, defined by $N^{2}=-\left(g / \rho_{0}\right) \mathrm{d} \bar{\rho} / \mathrm{d} z$, where $g$ is the gravitational acceleration, $\rho_{0}$ is a reference density and $\rho_{0}+\bar{\rho}(z)$ is the non-hydrostatic density profile, $z$ being the vertical coordinate oriented positively upwards. Their model is linear and implicitly assumes the upper and lower layers to be infinite. It describes the evolution of a plane internal gravity wave reflecting at the interface from above. It predicts a phase shift between the incident and reflected waves as well as the amplitude of the interfacial displacement induced by the reflection of the wave. This displacement was found to be largest for a specific density jump $\Delta \rho$ such that

$$
\beta \equiv g \frac{\Delta \rho}{\rho_{0}} \frac{k_{x}}{\omega^{2}} \simeq 1,
$$

where $k_{x}$ is the horizontal wavenumber component of the incident wave and $\omega$ is its frequency. This relation represents the square of a ratio of two phase speeds. First, 
the horizontal phase speed of the incident plane wave $\left(\omega / k_{x}\right)$. Second, the theoretical short-wave phase speed of interfacial waves (the upper and lower layers being of infinite extent) is given by $\left(g \Delta \rho /\left(2 k_{x} \rho_{0}\right)\right)^{1 / 2}$ and, apart from a factor of two, this expression features in (1.1). In Delisi \& Orlanski (1975), no reference was made to possible generation 89 of ISWs along the interface; instead the emphasis was on the occurrence of overturning.

Following the work of New \& Pingree in the 1990s, the theoretical model of Delisi \& Orlanski (1975) was considered again by Thorpe (1998). Thorpe derived the dispersion relation of interfacial waves for the case in which the homogeneous layer is finite. He investigated the influence of weakly nonlinear effects upon the reflected internal gravity wave and showed that a harmonic wave can be generated. He also discussed for what range of parameter values, as encountered in the Bay of Biscay, a generalised version of (1.1) could be satisfied; he argued that these would form favourable conditions for the development of ISWs in the pycnocline due to an incident internal tidal wave beam.

This work was further extended by Akylas et al. (2007), in the limit of long waves (in the sense that $k_{x} h \ll 1$, i.e. long with respect to the mixed layer thickness implying that non-hydrostatic effects are weak) and when an internal wave beam (instead of a simple plane wave of infinite horizontal extent) impinges on the pycnocline. Akylas et al. (2007) first addressed the linear development of the interfacial waves. Because the frequency of these waves is the forcing frequency $\omega$, they radiate energy back into the stratified fluid. Long-living interfacial waves are therefore prohibited in the linear regime. The weakly nonlinear regime was then investigated, the near-field and farfield evolutions leading to distinct solutions. In the near-field evolution, no radiation occurs and the interfacial displacement is found to be maximum when the wavelength of the interfacial wave matches the horizontal width of the incoming wave beam. The far-field evolution is described by an equation which involves weakly nonlinear, weakly non-hydrostatic and radiative effects. It admits soliton-like solutions obtained numerically.

The result of Akylas et al. (2007) confirmed the general idea behind 'local generation' as put forward by Gerkema (2001), namely that the initial phase is essentially linear and amounts to a 'scattering' of the beam as it encounters the pycnocline; nonlinear (and non-hydrostatic) effects become important in the second phase, when the perturbation of the pycnocline propagates away, steepens and may break up into ISWs. In some ways, the setting adopted by Gerkema (2001) was different from that of Akylas et al. (2007). In the former, too, the stratification consists of a mixed upper layer, an interfacial pycnocline and a constantly stratified lower layer, but the ocean depth was taken finite and the internal tidal beam (which impinges on the pycnocline from below) was generated by including barotropic tidal flow over infinitesimal topography. The linear problem was solved analytically in terms of vertical modes. All parameters were representative of the oceanic configuration of the Bay of Biscay and were fixed, except for the density jump across the interface, which was varied. It was then shown that the amplitude of the interfacial displacement is controlled by the ratio of the phase speed of interfacial long waves in a two-layer fluid to the phase speed of the first internal gravity wave mode in the uniformly stratified lower layer. It was concluded that the density jump across the pycnocline has to be moderate for the displacement amplitude of the interface to be maximum. If the density jump is stronger (than moderate), the beam is reflected at the interface. If the density jump is weaker, the beam is transmitted across the discontinuity as an evanescent wave whose decay length is much longer than the thickness of the 
homogeneous layer. In the Bay of Biscay indeed, typical horizontal wavelength of the beam is $10 \mathrm{~km}$ and the thickness of the upper mixed layer is about $50 \mathrm{~m}$. The beam is then reflected at the free surface and almost fully transmitted back into the stratified layer. Gerkema (2001) also derived and solved numerically the equations for the weakly nonlinear and weakly non-hydrostatic regime. Only for a moderate density jump was the nonlinear development of interfacial waves observed, evident from steepening and disintegration into ISWs. This work was extended by Maugé \& Gerkema (2008) to a more general setting in which the topography (here a continental slope) was allowed to be of finite amplitude.

All these studies are limited as they address, at best, weakly nonlinear and weakly non-hydrostatic effects and treat the pycnocline as an interface, i.e. a layer of infinitesimal thickness, with the exception of Maugé \& Gerkema (2008), who used stratification profiles obtained from measurements in the Bay of Biscay. In the present paper, we present numerical results obtained with the MIT general circulation model $($ MIT- $\mathrm{gcm})$; we are thus able to relax all these constraints at once: we allow a finite thickness of the pycnocline as well as fully nonlinear and non-hydrostatic effects. Our aim is to investigate when ISWs can be obtained in this more general situation, what is the influence of the finite thickness of the pycnocline on the wave structure and whether the criteria of Delisi \& Orlanski (1975), Gerkema (2001) and Akylas et al. (2007) for maximum pycnocline displacement still hold. The numerical set-up we consider is idealised, being two-dimensional, with simplified structures of stratification and forcing. Rotation is absent and the values of the parameters considered are inspired from laboratory experiments performed on the same problem in Grenoble. This experimental work will be reported in due time by their authors.

The numerical set-up is described in the next section. In $\S 3$, we show that a mode-1 ISW, which is the kind most commonly observed and documented, can be generated at the pycnocline by the impinging beam. A criterion for the selection of a particular mode is put forward for this purpose, in terms of phase speeds. This allows us to show in $\S 4$ that initial and forcing conditions can be designed such that mode- 2 or mode-3 ISWs are obtained. In addition, we propose in $\S 5$ another simple physical model to understand the selection of modes in the very near-field of the internal wave beam impact. The bandwidths of the selection criteria thus derived are discussed in $\$ 6$ along with the predictions of these criteria for the configuration of the Bay of Biscay. We conclude in $\S 7$.

\section{Numerical set-up}

We use the MIT-gcm code, a finite-volume, nonlinear, non-hydrostatic numerical model which solves the equations of motion under the Boussinesq approximation (Marshall et al. 1997). No subgrid modelling option is activated in our case, implying that the numerical simulations are direct.

We define a two-dimensional Cartesian coordinate system $(O, x, z)$ whose origin is at the top left-hand corner of a rectangular domain, with $z$ oriented vertically upwards. The Coriolis frequency is set to zero (for a discussion of effects of rotation, see \$6.2). An internal wave beam is imposed on the left boundary of the domain and propagates in a uniformly stratified fluid before impinging on a pycnocline of finite thickness. This configuration is described in detail below and sketched in figure 1. Values of all parameters defined in this section are displayed in tables 1 and 2.

The density profile we consider is continuous and consists of a homogeneous upper layer, a uniformly stratified lower layer and a pycnocline in between, namely a 
Brunt-Väisälä frequency in the lower layer

Forcing frequency

Forcing period

Angle of the internal wave beam in the lower layer

Vertical location of the centre of the pycnocline

Thickness of the pycnocline

Kinematic viscosity

Mass diffusivity

$\begin{array}{cl}N_{0} & 0.6 \mathrm{rad} \mathrm{s}^{-1} \\ \omega_{0} & 0.424 \mathrm{rad} \mathrm{s}^{-1} \\ T_{0}=2 \pi / \omega_{0} & 14.81 \mathrm{~s} \\ \theta_{0} & 45^{\circ} \\ h_{p} & 2 \mathrm{~cm} \\ \delta_{p} & 1 \mathrm{~cm} \\ v & 10^{-7} \mathrm{~m}^{2} \mathrm{~s}^{-1} \\ \kappa & 1.43 \times 10^{-9} \mathrm{~m}^{2} \mathrm{~s}^{-1}\end{array}$

TABLE 1. Set-up parameters that are common to all experiments.

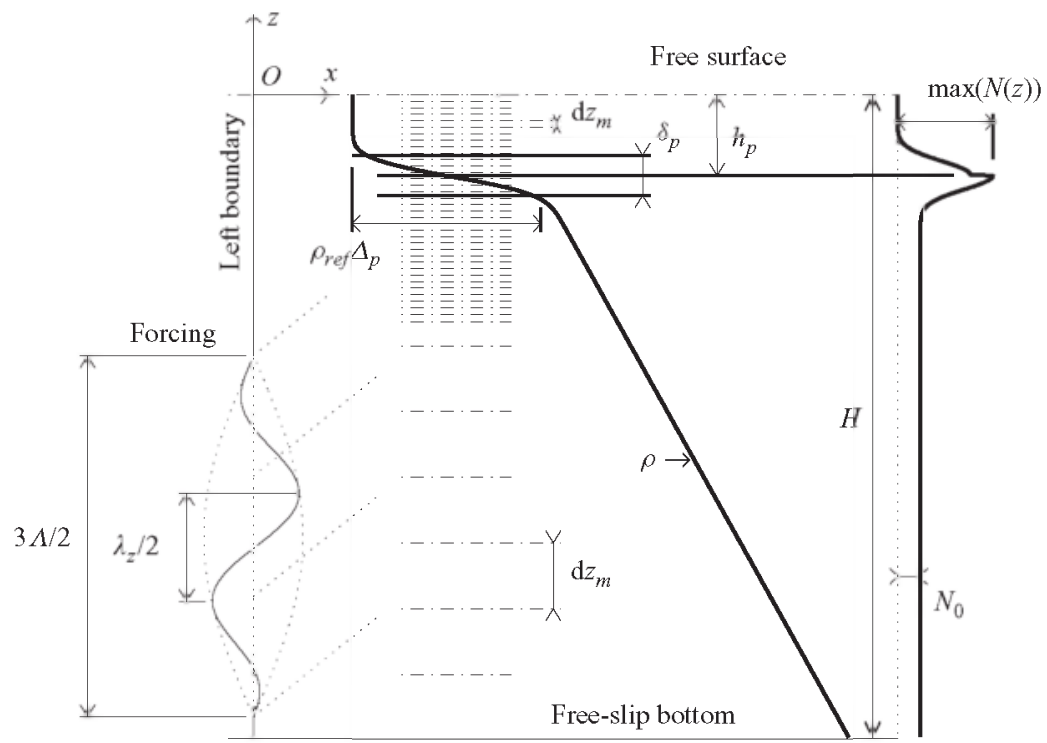

Figure 1. Sketch summarising the main features of the numerical set-up. Boundary conditions are indicated. The thick solid lines and bold labels refer to the density and Brunt-Väisälä profiles, as indicated. The horizontal dash-dotted lines and associated labels refer to the vertical setting of the grid. The coordinate system $(O, x, z)$ is sketched in the top left-hand corner. The dotted, sloping lines starting at the left boundary sketch internal wave characteristics of the beam.

strong but continuous change in density. The associated profile of the Brunt-Väisälä frequency is defined by

$$
N^{2}(z)=\frac{2}{\sqrt{\pi}} g \frac{\Delta_{p}}{\delta_{p}} \exp \left[-\left(\frac{z+h_{p}}{\delta_{p} / 2}\right)^{2}\right]+ \begin{cases}N_{0}^{2}, & \text { for }-H \leqslant z<-h_{p}, \\ 0, & \text { for }-h_{p} \leqslant z \leqslant 0,\end{cases}
$$

where $\Delta_{p}, \delta_{p}$ and $h_{p}$ are the relative change in density across the pycnocline, the thickness of the pycnocline and the depth of its centreline respectively, $H$ is the total water depth and $N_{0}$ is the (constant) Brunt-Väisälä frequency of the lower layer. Note that the initial profile $N^{2}(z)$ is not continuous, the discontinuity being however smoothed out by diffusion over a few forcing periods. No noticeable modification of $N^{2}(z)$ is to be seen over that time however.

In order to control the beam characteristics, we directly impose the wave beam at the left boundary of the domain in the lower layer (hence we do not model the generation 
Designation of the experiment

$\begin{array}{lccc} & \mathrm{E} 1 & \mathrm{E} 2 & \mathrm{E} 3 \\ H(\mathrm{~m}) & 0.95 & 0.8 & 0.8 \\ L(\mathrm{~m}) & 6 & 3 & 1.2 \\ A(\mathrm{~cm}) & 1.5 & 1.5 & 1.1 \\ \Lambda(\mathrm{cm}) & 60 & 26 & 15 \\ \lambda_{x}, \lambda_{z}(\mathrm{~cm}) & 53.6 & 23.2 & 13.4 \\ \lambda_{0}=\lambda_{x} \sin \theta_{0}(\mathrm{~cm}) & 37.9 & 16.4 & 9.48 \\ R e=\Lambda A \omega_{0} / \nu\left(\times 10^{5}\right) & 3.8 & 1.6 & 0.7 \\ 2 \pi A \sin \theta_{0} / \lambda_{z} & 0.12 & 0.29 & 0.37 \\ \Delta_{p}(\%) & 2.05 & 3.38 & 4 \\ \max N(z) / \omega_{0} & 11.2 & 14.3 & 15.5 \\ c^{*}=\sqrt{g \Lambda_{p} h_{p}}\left(\mathrm{~cm} \mathrm{~s}^{-1}\right) & 6.34 & 8.14 & 8.86 \\ \alpha=N_{0} \lambda_{0} / c^{*} & 4.0 & 1.6 & 0.96 \\ \gamma=c^{*} / N_{0} H & 0.11 & 0.17 & 0.19 \\ & 16 T_{0} & 12 T_{0} & 12 T_{0} \\ T_{0} / \mathrm{d} t & 400 & 800 & 250 \\ \mathrm{~d} x(\mathrm{~mm}) & 4 & 2 & 1 \\ \mathrm{~d} z_{m}(\mathrm{~mm}) & 0.4 & 0.3 & 0.2 \\ \mathrm{~d} z_{M}(\mathrm{~mm}) & 4 & 4 & 3.5 \\ l_{s}(\mathrm{~m}) & 2 & 1 & 0.25\end{array}$

Domain depth
Domain length
Along-beam forcing amplitude
Vertical scale of the forcing
Effective wavelengths of the forcing
Transverse wavelength of the beam
Reynolds number of the beam
Wave steepness of the beam
Relative density jump
Maximum value of $N(z) / \omega_{0}$
Long-wave phase speed, two-layer case
$\alpha$ parameter (see Akylas et al. 2007 )
$\gamma$ parameter (see Gerkema 2001)
Duration of the experiments
Number of time steps per period
Horizontal resolution
Fine vertical resolution
Coarse vertical resolution
Sponge layer length

TABLE 2. Set-up parameters that vary from one experiment to another.

of the wave beam over topography, as in Gerkema 2001). According to the dispersion relation of internal gravity waves, the energy of the wave beam propagates with the group velocity making an angle $\theta_{0}=\sin ^{-1}\left(\omega_{0} / N_{0}\right)$ with respect to the horizontal, where $\omega_{0}$ is the frequency of the wave beam (e.g. Lighthill 1978). We denote by $T_{0}$ the corresponding forcing period. The velocity profile of this wave beam is

$$
\boldsymbol{v}(x=0, z, t)=\Pi(z) \cos \left(\frac{2 \pi z}{\Lambda}+\omega_{0} t\right) \cos \left[\frac{2 \pi}{3 \Lambda}\left(z+\frac{H}{2}\right)\right] \boldsymbol{e}_{0},
$$

with

$$
\Pi(z)= \begin{cases}A \omega_{0}, & \text { for }|z+H / 2| \leqslant 3 \Lambda / 4 \\ 0, & \text { otherwise. }\end{cases}
$$

Here, $A$ is the amplitude of the along-beam displacement of particles located on the left boundary and $\boldsymbol{e}_{0}=\left(\cos \theta_{0}, \sin \theta_{0}\right)$ is the along-beam unit vector. This profile is displayed on the left-hand side of figure 1. As shown in (2.3), the forcing is applied on the vertical scale $3 \Lambda / 2$, which should therefore be smaller than the thickness of the uniformly stratified lower layer. This accounts for the total water depth $H$ to be slightly larger for experiment E1 than for experiments E2 and E3 (see table 2).

The profile (2.2) is a simple model of the far-field velocity profile of the internal wave field emitted by an oscillating object, the corresponding exact theoretical expression has been derived by Thomas \& Stevenson (1972). Here, the object would be the bathymetry of the continental shelf at the location of critical slope (Gostiaux \& Dauxois 2007; Zhang, King \& Swinney 2007). As shown by Staquet et al. (2006), a wave vector can be locally defined within the wave beam, though a wave beam is not a simple plane wave. This accounts for the profile (2.2) to be considered as spatially monochromatic. The profile (2.2) was also designed such that its integrated flux at the left boundary is always zero. In other words, it ensures that the free-surface mean displacement is zero at each time, which greatly improves the stability of the simulations. 
The effective horizontal and vertical wavelengths of the wave beam, denoted $\lambda_{x}$ and $\lambda_{z}$ respectively, can be inferred from (2.2), by calculating the distance $l$ between the maximum and minimum values of $|v|$ at a given time and defining $\lambda_{z}$ as $2 l$ and $\lambda_{x}$ as $2 l / \tan \theta$. From the values of $A$ and $\lambda_{z}$, the steepness of the waves defined by Thorpe (1987) as the amplitude of the vertical displacement of the isopycnals multiplied by the vertical wavenumber can be computed. The values are displayed in table 2 and never exceed 1, implying that the beam is statically stable in all three experiments.

Three numerical experiments denoted E1, E2 and E3 were carried out, each of which, as we shall see, is designed so that ISWs develop in the pycnocline with a different modal structure along the vertical. The length and frequency scales in tables 1 and 2 were taken from the laboratory experiments. The choice of the parameter $\Delta_{p}$ for experiment E1 (displayed in table 2) was guided by the numerical simulations of Gerkema (2001). The choice of the other parameters for this experiment is explained in $\S 4$, as well as those for experiments E2 and E3. Note that the length scale $\Lambda$ in (2.2) varies from one experiment to the other (for computational reasons), implying that the length of the domain $L$ varies as well to allow the pycnocline wave (evolving into a train of ISWs) to propagate over long enough distances. In the following, a pycnocline wave refers to an interfacial wave propagating in a pycnocline of finite thickness.

At the right end of the domain, a sponge layer of length $l_{s}$ is implemented to absorb the beam and the ISWs. This sponge layer consists of adding to the momentum equations an additional term characterised by a relaxation time scale, which forces the motions to evolve from their values at the boundary of the sponge layer facing the interior of the domain (or inner boundary) to the value prescribed at the end of the domain (zero in our case). In order to avoid reflections at the inner boundary back to the interior of the domain, the relaxation time is progressively decreased from $T_{0}$ at the inner boundary to $T_{0} / 1000$ at the boundary of the domain. This is the sponge layer implemented by default in the MIT-gcm which corresponds to the Herbaut formulation as described in Zhang \& Marotzke (1999).

The value of the viscosity $v$ was set to $10^{-7} \mathrm{~m}^{2} \mathrm{~s}^{-1}$. As we show below, this value ensures that mode-3 ISWs develop with significant amplitudes (for a realistic value of $v=10^{-6} \mathrm{~m}^{2} \mathrm{~s}^{-1}$, this mode is hardly visible and for $v \leqslant 10^{-8} \mathrm{~m}^{2} \mathrm{~s}^{-1}$, spurious effects occur at the smallest scales because of insufficient damping of those scales).

We use a grid with a constant horizontal resolution $\mathrm{d} x$ and with a vertical resolution that varies smoothly from a coarse resolution $\mathrm{d} z_{M}$ in the lower part to a fine resolution $\mathrm{d} z_{m}$ in the upper part of the domain, the middle of the transition zone being set to $z=-3 h_{p}$.

\section{Observations on the local generation of mode-1 ISWs}

\subsection{Preliminary considerations}

In the works of Gerkema (2001) and Akylas et al. (2007) mentioned in the Introduction, the phase speed of the interfacial waves generated as the beam impacts the pycnocline is characterised by the phase speed of long waves propagating at a pycnocline between two homogeneous fluids, namely $c^{\star}=\sqrt{g \Delta_{p} h_{p}}$, using notations of the previous section. In these works, the thickness of the pycnocline is infinitesimal, i.e. an interface.

At the region of beam impact, when the regime is still linear, Gerkema (2001) showed from analytical solutions that the parameter, denoted $\gamma$, controlling the displacement amplitude of the interface is the ratio of $c^{\star}$ over the phase speed of 
the first internal wave mode that propagates in the lower layer, $N_{0} H / \pi$. Thus, it was found that $\gamma=c^{\star} / N_{0} H$, ignoring a factor $\pi$ in front of $c^{\star}$. Gerkema (2001) found that values of $\gamma$ around $\simeq 0.12$ lead to the largest interfacial wave amplitudes for the oceanic configuration he considered.

In Akylas et al. (2007), the displacement amplitude of the interface was found to depend upon a parameter, denoted $\alpha$, comparing the horizontal width of the beam with the horizontal wavelength of the interfacial wave $2 \pi c^{\star} / \omega_{0}$ (since at the beam impact, the interfacial wave has the same frequency as the wave beam). In the present case, $\lambda_{x}$ is a good approximation of the beam horizontal width, so $\alpha$ can be expressed as $\alpha=N_{0} \lambda_{0} / c^{\star}$, introducing the transverse wavelength of the beam $\lambda_{0}=\lambda_{x} \sin \theta_{0}$ and ignoring again a factor $2 \pi$ in front of $c^{\star}$. Akylas et al. (2007) showed from numerical solutions of their soliton equation that maximum interfacial displacements occur when $\alpha \simeq 1$.

A third criterion was derived by Delisi \& Orlanski (1975) for short waves, which is (1.1). This criterion is nearly identical to that in terms of $\alpha$ mentioned above, except that the expression of the phase velocity of short interfacial waves propagating between two homogeneous fluids, which is $g \Delta_{p} /\left(2 k_{x}\right)$, is now used. This criterion will not be discussed further.

To sum up, the works of Akylas et al. (2007) and Gerkema (2001) lead to two parameters $\alpha$ and $\gamma$ which control the displacement amplitude of long interfacial waves, defined as

$$
\alpha=\frac{N_{0} \lambda_{0}}{c^{\star}}, \quad \frac{1}{\gamma}=\frac{H N_{0}}{c^{\star}} .
$$

These parameters are actually similar since they compare the phase speed $c^{\star}$ of an interfacial long wave between two homogeneous fluids with a typical phase speed of the forcing wave. This is the horizontal phase speed of the wave beam in Akylas et al. (2007) and the phase speed of its first mode in Gerkema (2001). The criteria based on $\alpha$ and $\gamma$ both ensure that the impinging of the internal wave beam upon the interface leads to its maximum displacement.

These parameters have been designed in the context of an infinitely thin pycnocline, hence supporting only mode-1 ISWs. As we will see in the following sections, only in E1 do mode-1 ISWs develop, and therefore only for E1 are $\alpha$ and $\gamma$ relevant. For purposes of illustration however, the values of $\alpha$ and $\gamma$ for all three experiments are displayed in table 2.

\subsection{Generation of mode-1 internal solitary waves}

Results from experiment E1 are displayed in figure 2, through contours of a few isopycnals around the pycnocline (figure $2 a$ ) and through the spatial distribution of the horizontal velocity field over the whole water depth (figure $2 b$ ). This figure displays two striking features. Figure $2(a)$ shows that a pycnocline wave is generated as the internal wave beam, visible in figure $2(b)$, hits the pycnocline. This wave degenerates into mode-1 ISWs. Figure 2(b) shows that the beam is strongly affected by the interaction with the pycnocline. A part of the beam is reflected downwards at the base of the pycnocline, while the remaining part is transmitted into the pycnocline. This transmitted part is refracted because the local Brunt-Väisälä frequency is stronger in the pycnocline; it reflects downwards on the upper boundary of the pycnocline and is partly transmitted back into the lower layer, thus emerging further away than the part that reflected at the base of the pycnocline (see Mathur \& Peacock 2009 for a detailed study of this process). Hence, the incident beam decomposes into two beams after reflecting from the pycnocline, resulting in the spreading of the beam 

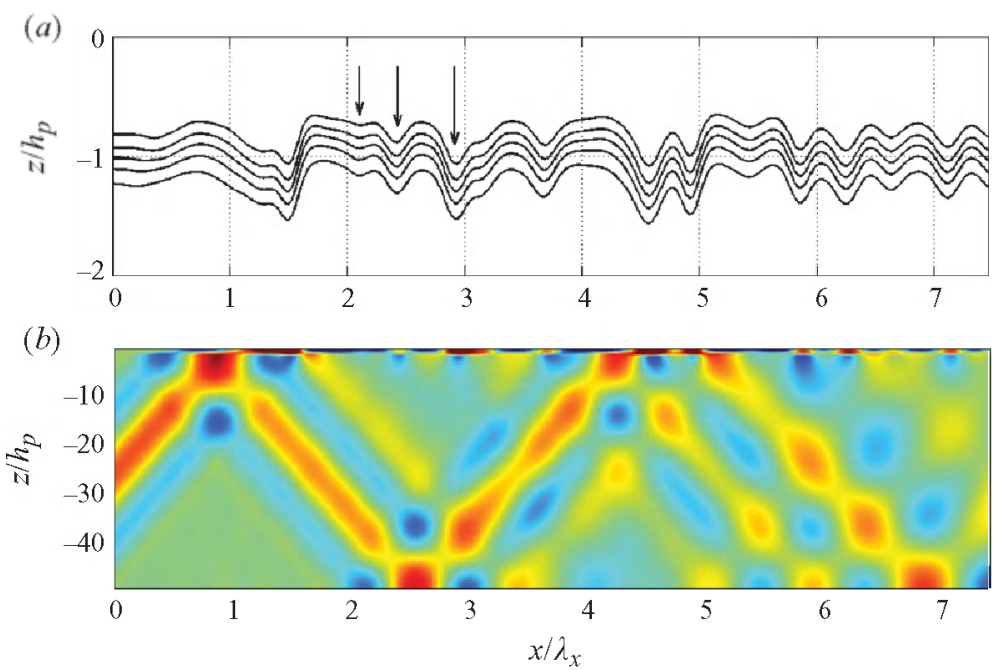

Figure 2. Generation of a mode-1 ISW by an internal wave beam in experiment E1. (a) Magnification of the isopycnals around the height $z=h_{p}$, with a train of three deformations explicitly indicated by arrows (the leading deformation has almost passed by the rear deformation of the preceding train). (b) Horizontal velocity field allowing to locate the internal wave beam; values range from $-7.5 \mathrm{~mm} \mathrm{~s}^{-1}$ (blue) to $7.5 \mathrm{~mm} \mathrm{~s}^{-1}$ (red).

energy in the lower layer. Gerkema (2001) also concluded from his linear solutions that 'the internal-beam energy gets spread all over the domain'. Note also that the Eulerian mean flow induced by the wave beam is weak, being at most $20 \%$ of the beam phase velocity at the impact zone and decaying downstream, with an associated Richardson number much larger than 1. This Eulerian drift will therefore not be considered hereafter.

The values of $\alpha$ and $\gamma$ defined by (3.2) have been computed for E1. We found $\alpha=3.6$, which is of the order of 1 as predicted by Akylas et al. (2007), and $\gamma$ equal to 0.11 , which is close to the value obtained by Gerkema (2001). This shows that, although the expressions of $\alpha$ and $\gamma$ rely on approximations (the pycnocline wave velocity is approximated by its long-wave expression between homogeneous fluids, the non-hydrostatic approximation is used and the thickness of the density jump is zero), these parameters are good indicators to predict whether optimal conditions are met for ISW generation.

The temporal development of the nonlinear dynamics of the pycnocline wave is displayed in figure 3 via space-time and space-frequency diagrams. Figure 3(a) displays the displacement of the isopycnal located in the middle of the pycnocline in a distance-time diagram. The horizontal axis is scaled by the horizontal effective wavelength of the incoming beam $\lambda_{x}$, while the unit of the vertical axis is the forcing period $T_{0}$. In figure $3(b)$, the power spectral density of this displacement is displayed in a distance-frequency diagram. The vertical axis is scaled by the forcing frequency $\omega_{0}$ for a clearer detection of harmonic frequencies of $\omega_{0}$ by nonlinear effects.

Figures 2 and 3 show that the pycnocline dynamics can be decomposed into three stages. (i) As noted above, the beam impinges on the pycnocline and part of its energy is transmitted into the pycnocline, the remaining part being reflected (figure $2 b$ ). The transmitted beam excites a pycnocline wave and this generation process is essentially linear, as argued earlier by Gerkema (2001) and Akylas et al. 

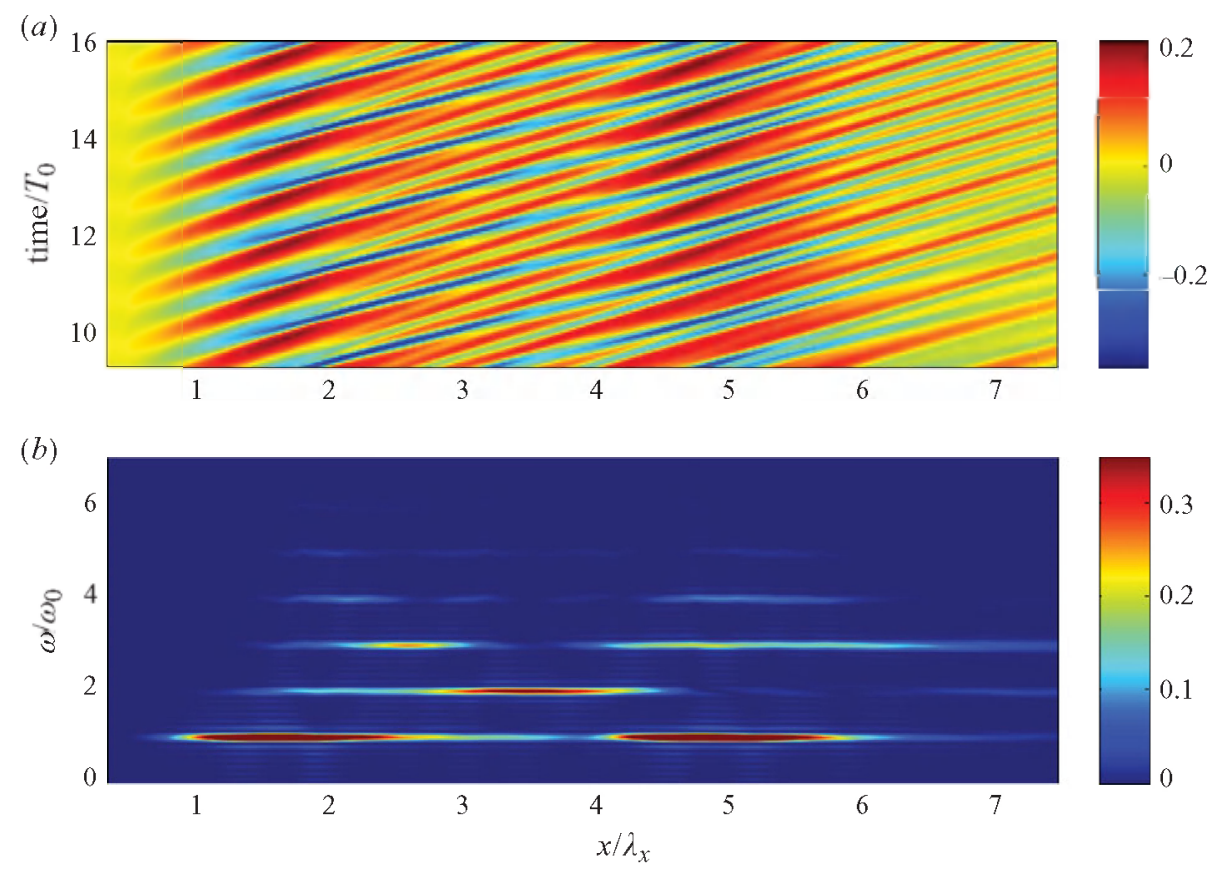

FIgURE 3. Temporal analysis of the pycnocline displacement for E1. (a) The $(x, t)$ plot of the vertical displacement of the isopycnal located at the centre of the pycnocline (whose depth at rest is $z=-h_{p}$ ). The vertical displacement is scaled by $h_{p}, T_{0}$ is the period of the forcing beam, and $\lambda_{x}$ is the effective horizontal wavelength of that beam. $(b)$ The $(x, \omega)$ plot of the power spectral density of $(a)$, computed as the squared modulus of the temporal Fourier transform of the pycnocline displacement and scaled by its maximum value.

(2007). As shown in figure $3(b)$, indeed, the dominant frequency for $x / \lambda_{x} \lesssim 1$ is the forcing frequency. (ii) Approximately one $\lambda_{x}$ further, i.e. for $1 \leqslant x / \lambda_{x} \lesssim 2$, this pycnocline wave propagates horizontally and steepens, a nonlinear effect. Such a steep front can be seen in figure 2(a), at location $x / \lambda_{x}=1.5$. The growing importance of nonlinearity is also evident from figure $3(b)$, which shows that higher harmonics of $\omega_{0}$ are being developed during this stage. Since $2 \omega_{0} \approx 0.8 \mathrm{rad} \mathrm{s}^{-1}>N_{\mathrm{c}}$. the now nonlinear pycnocline wave remains trapped in the pycnocline and grows in amplitude due to the continuous internal wave beam forcing. (iii) Finally, further away (for $x / \lambda_{x} \geq 2$ ), the large amplitude pycnocline wave disintegrates into trains of three shorter, amplitude-ordered and solitary-like deformations. This is indicated by arrows in figure 2(a). The largest deformations propagate faster than the smallest ones, hence the amplitude ordering. This can be seen in the pattern in figure 3(a), for example for $2<x / \lambda_{x}<4$, where the different propagation speeds manifest themselves in different slopes of the lines following the deformations.

These ISWs eventually decay, possibly because of viscosity, although radiation of internal waves in the lower stratified layer might play a role. The latter phenomenon is actually visible in figure $2(b)$, where a low-amplitude signature of radiated waves outside the main envelope of the beam can be detected close to the pycnocline. To rule out the possibility of a viscous decay, let us introduce the $e$-folding viscous decay length scale of the amplitude, denoted $\xi$. Assuming that the ISW may be modelled as an internal gravity wave of frequency $\hat{\omega}>N_{0}$ and horizontal wavenumber $\hat{k}$ propagating in the pycnocline of the Brunt-Väisälä frequency $\hat{N}, \xi$ is given by 
(e.g. Lighthill 1978, p. 272)

$$
\xi=\frac{1}{v}\left(\frac{\hat{\omega}}{\hat{k}}\right)^{3} \frac{\sqrt{\hat{N}^{2}-\hat{\omega}^{2}}}{\hat{N}^{3}} .
$$

The ratio $\hat{\omega} / \hat{k}$ is the horizontal phase velocity of the wave and, as we shall see in the next section, is well approximated by the horizontal phase velocity of the beam $\omega_{0} / k_{x}$. The time $2 \pi / \hat{\omega}$ is the typical temporal width of the ISW and, from figure $3(a)$, can be estimated as $T_{0} / 3$. Using $\hat{N}=4 \mathrm{rad} \mathrm{s}^{-1}$ as a typical measure of the BruntVäisälä frequency in the pycnocline, we get $\xi=28 \mathrm{~m} \approx 52 \lambda_{x}$, which is much larger than the length of the domain $L=6 \mathrm{~m} \approx 11 \lambda_{x}$. Hence, viscosity can be ignored in this experiment.

\section{How to control the mode number: a 'far-field' approach}

\subsection{Heuristic considerations: the modal decomposition}

In the present case of a pycnocline of finite thickness, the modal decomposition provides a simple method to study the structure and characteristics of the full internal gravity wave field that develops over the total water depth. We shall show in this section that this method also provides a useful heuristical tool to design a numerical experiment so that mode-1 ISWs develop.

For the sake of simplicity, we will make a rigid-lid approximation. Indeed, in all our experiments, the free-surface displacement never exceeds $0.2 \mathrm{~mm}$, which is two orders of magnitude smaller than the thickness of the upper homogeneous layer $h_{p}$.

We briefly recall the principles of the modal decomposition (see e.g. Leblond \& Mysak 1978 for detail). When searching for a vertical velocity solution of the linear inviscid Boussinesq equations of the form

$$
w(x, z, t)=W(z) \exp \mathrm{i}(K x-\Omega t)
$$

with $K>0$ as we are only interested in rightward-propagating waves, it can be shown that $W$ and $K$ satisfy the eigenvalue problem:

$$
\begin{gathered}
\frac{\mathrm{d}^{2} W}{\mathrm{~d} z^{2}}+K^{2} \frac{N^{2}(z)-\Omega^{2}}{\Omega^{2}} W=0, \\
\left.W\right|_{z=0}=\left.W\right|_{z=-H}=0 .
\end{gathered}
$$

The system of $(4.2 a)$ and (4.2b) forms a Sturm-Liouville problem and has an infinite sequence of modes, of eigenfunctions $W_{n}$ associated with eigenvalues $K_{n}$ ( $n$ being the mode number). The general solution for $w$ limited to rightward-propagating components can therefore be written as

$$
w(x, z, t)=\sum_{n=0}^{\infty} a_{n} W_{n}(z) \exp \mathrm{i}\left(K_{n} x-\Omega t\right),
$$

where the coefficients $\left(a_{n}\right), n \in \mathbb{N}$, are the amplitude of the modes. Solving $(4.2 a)$ and (4.2b) then requires $N(z)$ and $\Omega$ as input parameters which determine $W_{n}$ and $K_{n}$ for each mode $n$ (setting $K$ and retrieving $\Omega_{n}$ would also have been possible). The phase speeds of each mode are then $c_{n}=\Omega / K_{n}$ (or $\Omega_{n} / K$ ).

The case of experiment E1 is now considered to illustrate how we use heuristically this method to determine the parameter set that favours the development of a mode-1 ISW. 


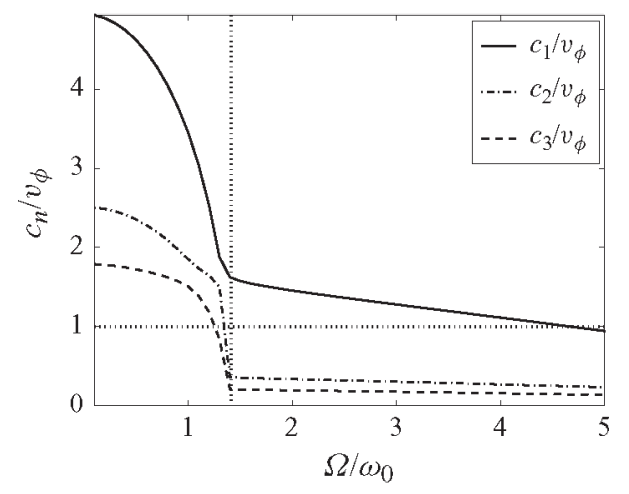

FIGURE 4. Phase speeds of the first three modes $c_{1}, c_{2}$ and $c_{3}$ for experiment E1 computed from (4.2a) and (4.2b) as a function of $\Omega$ and for $N(z)$ given by (2.1). The phase speeds and $\Omega$ are scaled by the phase speed $v_{\phi}$ and frequency $\omega_{0}$ of the wave beam, respectively. The vertical dashed line marks the location where $\Omega=N_{0}$.

In the work of Akylas et al. (2007), the maximum interfacial displacement is obtained when the horizontal width of the beam (which is well approximated by $\lambda_{x}$ in our case) matches the horizontal wavelength of the interfacial wave. Since this wave is forced at the beam frequency, its horizontal phase speed therefore matches the horizontal phase speed of the beam. As we now show, when the pycnocline is of finite thickness and weakly nonlinear effects have developed, the mode-1 pycnocline wave also has the same horizontal phase speed as the incoming beam.

To apply the modal decomposition to experiment E1, we assume that an ISW may be modelled as a superposition of linear internal gravity waves which are trapped within the pycnocline, i.e. of frequency comprised between $N_{0}$ and $\max (N(z))$. We solve (4.2a) and (4.2b) with $\Omega$ varying and display the phase speeds of the first three modes $c_{1}, c_{2}$ and $c_{3}$ versus $\Omega$ in figure 4 . The figure shows that the phase speed decreases as $\Omega$ increases, with a sharp transition when $\Omega=N_{0}$ (i.e. for $\Omega / \omega_{0}=\sqrt{2}$ ): the decrease rate of the phase speeds drops, corresponding to the modes being trapped in the pycnocline. For $\Omega \geqslant N_{0}$, all three phase speeds evolve quasi-linearly, with $\Omega$, $c_{2}$ and $c_{3}$ being nearly constant and distinct from $c_{1}$. The velocity $c_{1}$ is very close to the horizontal phase velocity $v_{\phi}$ of the wave beam as $\Omega$ increases.

Hence, to select a mode-1 ISW, the internal wave beam should be designed such that its horizontal phase speed is close to $c_{1}$ (computed from modal decomposition for $\Omega \in] N_{0}, \max (N(z))[, N(z)$ being given). This is how experiment E1 was designed, adjusting also the wave beam amplitude so that a strong deformation is induced in the pycnocline while ensuring that the beam remains stable.

We will now make the conjecture that in order to excite mode- $n$ ISWs, the horizontal phase speed of the internal wave beam should be within the range of values of the phase speeds of the mode- $n$ internal waves when trapped in the pycnocline (i.e. with frequency $\Omega \in] N_{0}, \max (N(z)$ ) [). This conjecture will be verified next.

\subsection{Application to the generation of mode-2 and mode-3 internal solitary waves}

We now focus on experiments E2 and E3. In figures $5(a)$ and $5(b)$, the phase speeds of the first three modes are plotted versus $\Omega$ for experiments E2 and E3, respectively. These phase speeds display the same behaviour as for experiment E1, $c_{2}$ and $c_{3}$ reaching a nearly constant value as soon as $\Omega$ exceeds $N_{0}$. We find that for $\Omega>N_{0}$, $v_{\phi}\left(\approx 1.57 \mathrm{~cm} \mathrm{~s}^{-1}\right)$ in experiment E2 is closer to $c_{2}\left(\approx 1.5 \mathrm{~cm} \mathrm{~s}^{-1}\right.$ as far as we computed 

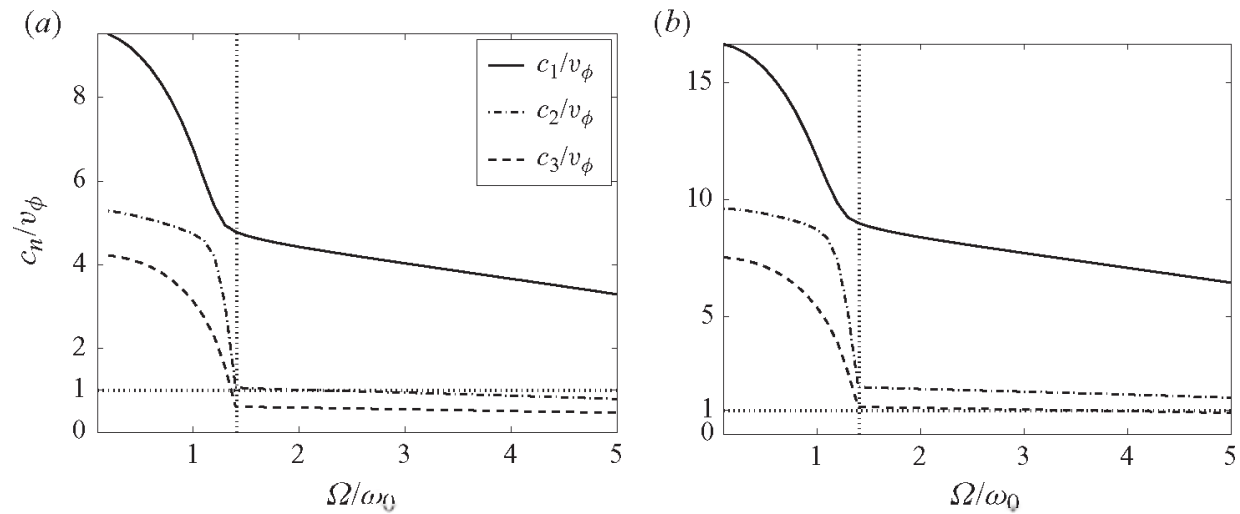

Figure 5. Same as in figure 4 but for experiments (a) E2 and (b) E3.

$(a)$

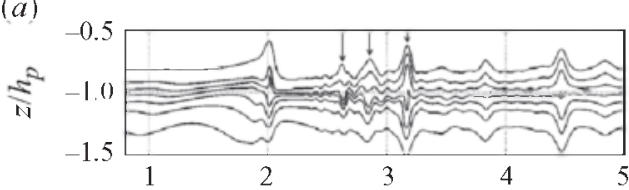

(b)

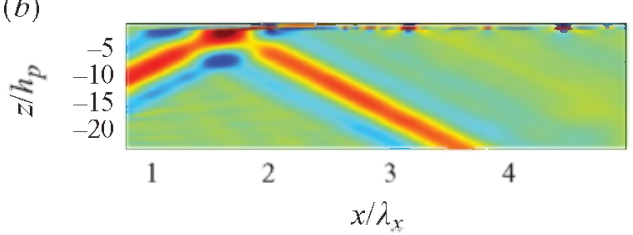

(c)

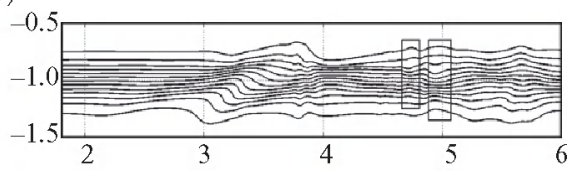

(d)

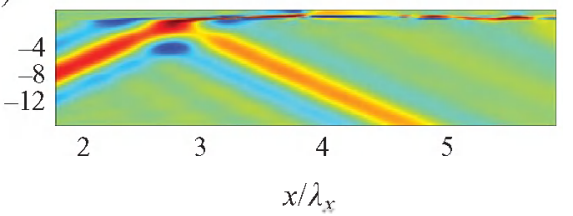

FIgURE 6. Generation of ISWs by an internal wave beam in experiments E2 and E3. This figure is similar to figure 2 for experiment E1. In $(a)$, mode-2 ISWs develop and a train with three visible deformations is highlighted with arrows. In (c), mode-3 ISWs are framed, consisting of a train with two visible deformations. In $(b)$, values range from $-5 \mathrm{~mm} \mathrm{~s}^{-1}$ (blue) to $5 \mathrm{~mm} \mathrm{~s}^{-1}$ (red) and in $(d)$, values range from $-4 \mathrm{~mm} \mathrm{~s}^{-1}$ (blue) to $4 \mathrm{~mm} \mathrm{~s}^{-1}$ (red).

it) in this case. When the modal decomposition is applied to E3, the value of $c_{3}$ for $\Omega>N_{0}\left(\approx 1 \mathrm{~cm} \mathrm{~s}^{-1}\right)$ here is the closest to $v_{\phi}\left(\approx 0.905 \mathrm{~cm} \mathrm{~s}^{-1}\right)$. Like E1 with $c_{1}$, experiments E2 and E3 were actually designed from the computation of $c_{2}$ and $c_{3}$ respectively, such that the horizontal phase speed of the wave beam matches $c_{2}$ in E2 and $c_{3}$ in $\mathrm{E} 3$.

Visualisations of the fields similar to figure 2 are displayed in figure 6 for each experiment. Figure 6 shows that ISWs develop again from the impact of the wave beam on the pycnocline (figure $6 b, d$ ). However, the vertical structure of the pycnocline wave is now a mode 2 for $\mathrm{E} 2$ (figure $6 a$ ) and a mode 3 in $\mathrm{E} 3$ (figure $6 c$ ), the number of visible deformations per train being three in the former case and two in the latter. The amplitude of the mode-3 ISWs is actually rather weak and, as we will see next, very sensitive to viscous effects. We verified that the three stages of the process going from a linear to a weakly nonlinear internal wave trapped in the pycnocline, described in $\$ 3.2$, also occur in these two experiments.

In order to validate a posteriori the value of $v$ we chose, we recall (3.2). For E2, using $2 \pi / \hat{\omega}=T_{0} / 8$ and $\hat{N}=5.5 \mathrm{rad} \mathrm{s}^{-1}$, we obtain $\xi=1 \mathrm{~m} \approx 4.3 \lambda_{x}$, which is now smaller than the length of the domain $\left(L=3 \mathrm{~m} \approx 13 \lambda_{x}\right)$. For experiment $\mathrm{E} 3$, using $2 \pi / \hat{\omega}=T_{0} / 6$ and 


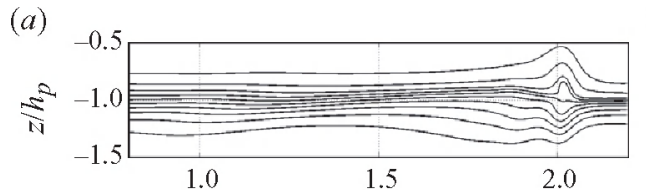

(b)

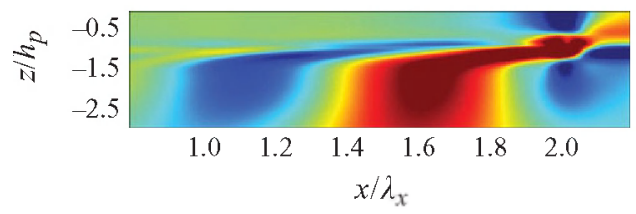

(c)

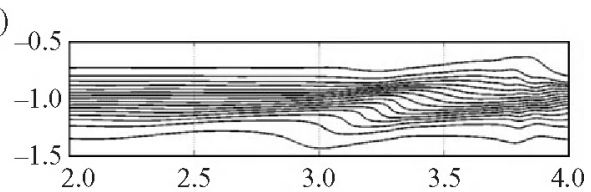

(d)

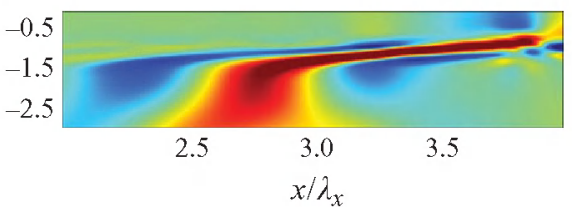

FIgURE 7. Same as in figure 6 but for experiments E2 $(a, b)$ and E3 $(c, d)$, magnified around the zones of impact of the internal wave beam in order to emphasise the refraction of the beam during its propagation in the pycnocline.

$\hat{N}=6 \mathrm{rad} \mathrm{s}^{-1}$ yields $\xi=0.18 \mathrm{~m} \approx 1.3 \lambda_{x}$, which is even smaller compared to $L$ (since $L=1.2 \mathrm{~m} \approx 9 \lambda_{x}$ ). The values of $\hat{\omega}$ for $\mathrm{E} 2$ and $\mathrm{E} 3$ have been estimated from figure 6 . The orders of magnitude for $\xi$ show that for E2 and E3, viscosity plays an important role in the decay of the ISWs. Hence, the use for $v$ of the realistic value $10^{-6} \mathrm{~m}^{2} \mathrm{~s}^{-1}$ would have led to a very quick attenuation of mode- 2 ISWs and prevented the development of mode-3 ISWs, as we verified.

\section{How to control the mode number: a 'near-field' approach}

The approach developed above addresses the 'far-field' evolution of the dynamics within the pycnocline, when nonlinear waves have developed. The approach we consider now may be referred to as the 'near-field' evolution, in the sense that we analyse the deformation of the pycnocline at the beam impact.

The simple model we shall derive is motivated by a careful inspection of the impact zone of the internal wave beam in experiments E2 and E3, which is magnified in figure 7 . The beam is refracted as it gets into the pycnocline, since its frequency and horizontal wavelength remain unchanged (the dynamics being linear and the changes of the medium occurring along $z$ only) whereas the local Brunt-Väisälä frequency increases. We shall see that conditions for optimal forcing of the pycnocline wave by the beam can be derived, which also set the vertical structure (i.e. mode number) of the pycnocline wave.

The model is based on three approximations: (i) we focus on the initial phase of wave generation in the pycnocline, so that the dynamics may be assumed linear; (ii) we also consider a simple plane wave instead of a wave beam; (iii) we assume that the profile of the Brunt-Väisälä frequency is a piecewise continuous function consisting of three parts, namely

$$
N_{3 L}^{2}(z)= \begin{cases}N_{0}^{2}, & \text { for }-H \leqslant z<-h_{p}-\frac{\delta_{p}}{2}, \\ N_{i}^{2}, & \text { for }-h_{p}-\frac{\delta_{p}}{2} \leqslant z \leqslant-h_{p}+\frac{\delta_{p}}{2}, \\ 0, & \text { for }-h_{p}+\frac{\delta_{p}}{2}<z \leqslant 0,\end{cases}
$$

where $N_{i}$ is the Brunt-Väisälä frequency within the pycnocline. The value of $N_{i}$ has been chosen in order to preserve the phase shift between the induced displacements 
(a)

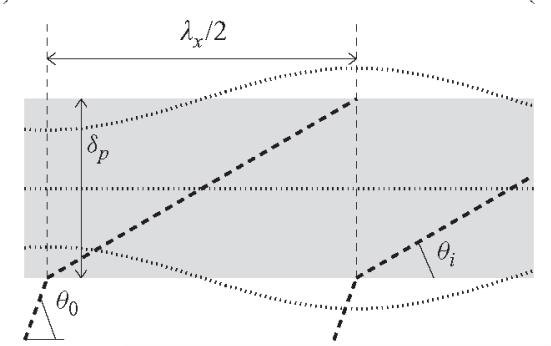

(b)

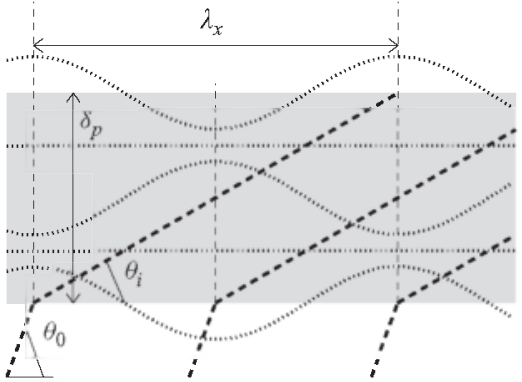

FIGURE 8. Sketches illustrating the conditions for the generation of mode-2 $(a)$ and mode- 3 (b) internal waves trapped in a pycnocline using the linear, three-layer model presented in $\$ 5$. The shaded area is the pycnocline, the thick dashed lines represent isophases of the displacement separated by a distance $\lambda_{x} / 2$ (phase shift $\pi$ ) and the dotted lines sketch the displacements induced by the plane wave in the pycnocline, highlighting the selected vertical structure.

of the top and bottom of the pycnocline. In other words, let us assume that in our numerical experiments, an internal wave characteristic impinges on the bottom of the pycnocline at a location $x_{0}$ and is refracted until being reflected against the top of the pycnocline at the location $x_{0}+\Delta x$. Our three-layer model is designed such that the locations $x_{0}$ and $x_{0}+\Delta x$ are preserved, although the characteristics are now piecewise straight lines. The slope of a characteristic is equal to $\sqrt{\omega_{0}^{2} /\left(N^{2}(z)-\omega_{0}^{2}\right)}$ and $\Delta x$ is computed by integrating the path of a characteristic between the boundaries of the pycnocline, which we set to be at depths $z_{1}=-h_{p}-\delta_{p} / 2$ and $z_{2}=-h_{p}+\delta_{p} / 2$ :

$$
\Delta x=\int_{z_{1}}^{z_{2}} \mathrm{~d} z \sqrt{\frac{N^{2}(z)-\omega_{0}^{2}}{\omega_{0}^{2}}} .
$$

In the case of a pycnocline characterised by the constant Brunt-Väisälä frequency $N_{i}$, this expression reduces to $\Delta x=\delta_{p} \sqrt{\left(N_{i}^{2}-\omega_{0}^{2}\right) / \omega_{0}^{2}}$. Matching these two expressions then leads to the following expression for $N_{i}$ :

$$
N_{i}^{2}=\omega_{0}^{2}+\frac{\omega_{0}^{2}}{\delta_{p}^{2}}\left(\int_{z_{1}}^{z_{2}} \mathrm{~d} z \sqrt{\frac{N^{2}(z)-\omega_{0}^{2}}{\omega_{0}^{2}}}\right)^{2} .
$$

We show that a Bragg-like resonance condition based on simple geometric arguments can be obtained for the selection of the mode. At depth $z=-h_{p}-\delta_{p} / 2$, upon entering the pycnocline, the angle of propagation of the energy of the plane wave is changed from $\theta_{0}$ to $\theta_{i}=\arcsin \left(\omega_{0} / N_{i}\right)<\theta_{0}$, due to refraction. Leaving aside the mode- 1 case, which does not seem to be tractable with the present approach, we now distinguish the two cases we investigate, starting with the forcing of a mode- 2 pycnocline wave.

Two isophases of the incident plane wave are plotted in figure $8(a)$ as well as two isopycnals at the top and bottom of the pycnocline. If the horizontal wavelength of the plane wave $\lambda_{x}$ is such that $\lambda_{x} / 2=\Delta x$, the induced displacements of the top and bottom of the pycnocline are opposite in phase (phase shift of $\pi$ ), making the vertical structure of the refracted wave in the pycnocline similar to that of a mode- 2 internal wave. Figure $8(a)$ only displays wave characteristics until one reaches the top of the pycnocline but the wave characteristics can be drawn further along the pycnocline without altering the pattern of a mode- 2 internal wave. The subsequent steps are 


$\begin{array}{lll}\text { Experiment } & \text { E2 } & \text { E3 } \\ n & 2 & 3 \\ N_{i}\left(\mathrm{rad} \mathrm{s}^{-1}\right) & 5.24 & 5.68 \\ \mu_{n} & 0.94 & 1.00\end{array}$

TABLE 3. Verification of relation (5.6) for experiments E2 and E3. Here $n$ is the mode number of the pycnocline wave, $N_{i}$ (defined by (5.3)) is the local Brunt-Väisälä frequency in the pycnocline and the parameter $\mu_{n}$ is the left-hand side of relation (5.6).

similar to those of any ISW generation: the isopycnals forming the symmetrical pattern, typical of a mode- 2 wave, steepen and split up into trains of mode-2 ISWs. Since $\Delta x=\delta_{p} / \tan \theta_{i}$, a mode-2 pycnocline wave is therefore forced if

$$
\frac{\lambda_{x} \tan \theta_{i}}{2 \delta_{p}}=1
$$

The case of a mode- 3 pycnocline wave is sketched in figure $8(b)$. The displacements of the top and bottom of the pycnocline have a phase shift of $2 \pi$ if $\Delta x=\lambda_{x}$, implying that within the pycnocline, the displacement of the middle isopycnal is opposite in phase with the displacements of the top and bottom ones, as for a mode-3 wave. Hence, a mode- 3 pycnocline wave is forced if

$$
\frac{\lambda_{x} \tan \theta_{i}}{\delta_{p}}=1
$$

The two conditions described here can be gathered into a single one and extended to any mode $n \geqslant 2$ by noting that the displacements of the top and bottom of the pycnocline should have a phase shift of $(n-1) \pi$ for the vertical structure of the wave to resemble a mode- $n$ internal wave trapped in the pycnocline. Conditions (5.4) and (5.5) can thus be generalised to any mode number $n(n \geqslant 2)$ as

$$
\frac{n-1}{2} \frac{\lambda_{x} \tan \theta_{i}}{\delta_{p}}=1
$$

The validity of relation (5.6) for the two experiments E2 and E3 is checked by computing the left-hand side of this relation, which we denote by $\mu_{n}$. The values of $\mu_{2}$ and $\mu_{3}$ are displayed in table 3: $\mu_{2}$ departs from the value of 1 by $6 \%$ and $\mu_{3}$ by less than $1 \%$, attesting that relation (5.6) is well satisfied for these two experiments. This relation explains why a beam with a short wavelength generates high-mode pycnocline waves or, equivalently, why such high-mode waves are favoured by a strongly stratified pycnocline.

Relation (5.6) has not been checked numerically for higher modes as the conditions to generate a displacement that is large enough for nonlinear effects occurring later are then much more difficult to achieve and would be associated with a costly increase in resolution. Note that this approach does not apply to mode-1 pycnocline waves. It relies indeed on the phase shift between the displacements along the top and bottom of the pycnocline, while a mode- 1 wave induces vertical displacements that are all in phase along the vertical. An indication however that mode-1 ISWs can be generated is when the value of $\mu_{2}$ is noticeably higher than one, since $\mu_{2}=1$ when mode- 2 ISWs are generated.

It is not clear to us how to relate this 'near-field' approach to the 'far-field' approach derived in the previous section. It is useful however to bring together the conclusions 
of the two approaches. Writing $v_{\dot{\phi}}=c_{n}$ on the one hand, and relation (5.6) on the other hand, allows one to derive an approximate analytical expression for $c_{n}$, namely

$$
c_{n}=\frac{\delta_{p} \sqrt{N_{i}^{2}-\omega_{0}^{2}}}{\pi(n-1)} .
$$

This is obtained by expressing (5.6) in terms of $v_{\phi}\left(=\omega_{0} \lambda_{x} / 2 \pi\right)$, writing the dispersion relation as $\omega_{0}=N_{i} \sin \theta_{i}$ and therefore $N_{i} \cos \theta_{i}=\sqrt{N_{i}^{2}-\omega_{0}^{2}}$. As $N_{i} \gg \omega_{0}$ for the three experiments, (5.7) can be simplified to $c_{n}=\delta_{p} N_{i} /(\pi(n-1))$. Numerical estimate of $c_{n}$ given by (5.7) for the parameters of table 2 yields $c_{2} \approx 1.66 \mathrm{~cm} \mathrm{~s}^{-1}$ for E2 and $c_{3} \approx 0.901 \mathrm{~cm} \mathrm{~s}^{-1}$ for $\mathrm{E} 3$, which is in good agreement with the values given in $\S 4.2$.

\section{Discussion}

\subsection{Bandwidths of the selection conditions}

This paper aims at determining optimal conditions to generate mode- $n$ ISWs. However, the bandwidths of these conditions have not been discussed. In other words, when for a given situation, $\mu_{n}<1<\mu_{n+1}$ and/or $c_{n}<v_{\phi}<c_{n+1}$ in the trapped regime, it is yet to be determined whether ISWs develop at all and, if they do, which of the two $n$ or $n+1$ modes would preferentially develop and what would be their amplitudes. From a qualitative point of view, the answer must depend on several parameters, such as the ratio $\left|c_{n}-v_{\phi}\right| /\left|v_{\phi}-c_{n+1}\right|$ in the trapped regime or any quantity comparing the distances between $v_{\phi}$ and the different phase speeds in that regime. From a quantitative point of view, the computation of the amplitude of the trapped modes would provide a precise answer but would be quite involved as the nonlinear nature of these waves implies that the motion cannot be easily projected on an orthogonal basis of linear wave modes at a given frequency. The projection of the motion on an orthogonal basis of nonlinear modes would require to know which model (such as a high-order KdV model) describes it accurately, which has not been done for the present paper.

To get some indication of the bandwidths of the selection conditions (i.e. of the near-field and far-field conditions), we rather performed another numerical experiment (not shown). This experiment involves a beam, for which $v_{\dot{\phi}}$ is the same as in E1 but the stratification profile is the same as in E2. From the near-field condition (5.4), one finds $\mu_{2}=2.17>1$, which implies that mode-1 ISWs can be generated (as the near-field condition does not apply to mode-1 ISWs, it is not possible to be more specific). Regarding the far-field condition, figure $5(a)$ for $\mathrm{E} 2$ shows that $v_{\phi}$ is halfway between $c_{1}$ and $c_{2}$ in the trapped regime (since $v_{\phi}$ for E1 is 2.3 times larger than for E2). Therefore, we are in an intermediate regime.

Observation of the pycnocline motion in this experiment shows a dominant mode-1 pattern for the ISWs, with an amplitude of the induced isopycnal displacement of about $3 \mathrm{~mm}$. Superposed on this isopycnal displacement is a secondary pattern, typical of mode-2 ISWs. Mode-2 isopycnal displacements (measured with respect to the displacements, already induced by the mode-1 ISWs) are less than $1 \mathrm{~mm}$. On the basis of this crude estimate, we infer that the two bandwidths for the far-field selection conditions overlap but the bandwidth for the selection of mode-1 ISWs seems to be larger than that for mode-2 ISWs.

Two conjectures can be proposed based on this example: first, that when in an intermediate case, ISWs can be generated and the bandwidths of the selection conditions for each mode number can overlap; second, that the lower the mode of 


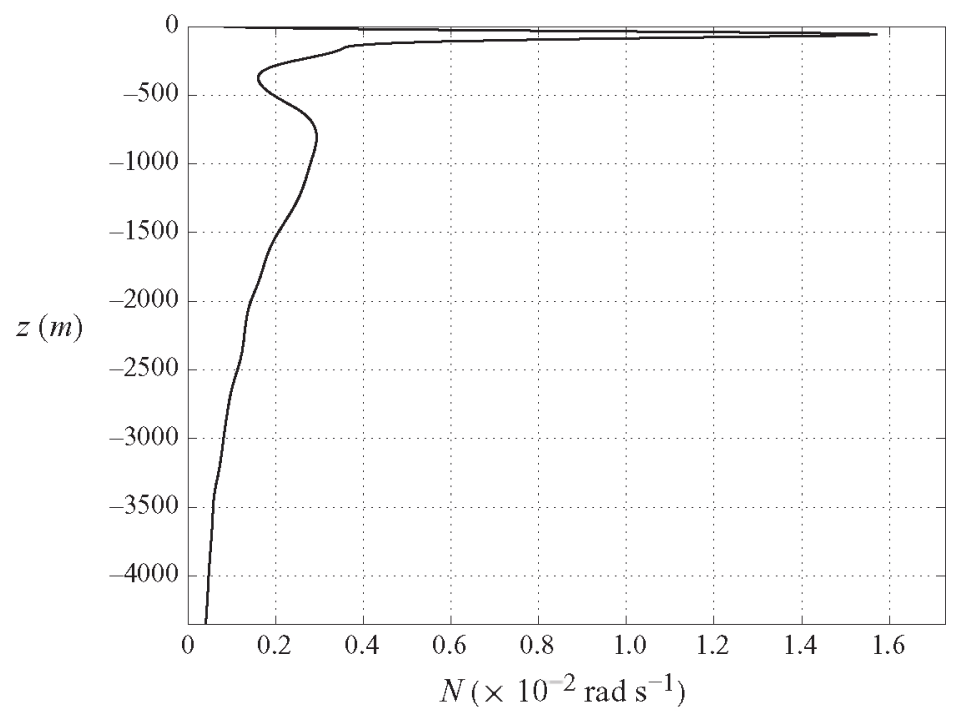

FIGURE 9. Typical stratification observed in the Bay of Biscay in mid-to-late summer.

the ISWs is, the broader the bandwidth of its selection condition might be. These conjectures both seemed to be verified in a few other numerical tests that helped us to derive our selection conditions.

\subsection{Applicability of the selection conditions to an oceanic setting}

This paper addresses fundamental aspects of the generation of ISWs by an internal wave beam. However, let us recall that this problem was motivated by oceanic observations. In this section, we apply the selection conditions to the more realistic context of the Bay of Biscay and investigate whether these conditions can correctly predict the observations. (As found by New \& Pingree 1990, 1992, local generation in the Bay of Biscay is associated with mode-1 ISWs.) We will also show that rotation can be easily introduced in the far-field and near-field approaches.

The reference stratification profile we consider is a typical profile observed in the Bay of Biscay in mid-to-late summer, when the seasonal pycnocline is best developed and when local generation is observed. This reference profile (displayed in figure 9) is characterised by a strong seasonal pycnocline whose maximal value is $1.6 \times 10^{-2} \mathrm{rad} \mathrm{s}^{-1}$ at $58 \mathrm{~m}$ depth. Note the presence of a permanent pycnocline corresponding to increased values of $N$ between roughly $400 \mathrm{~m}$ and $2000 \mathrm{~m}$ depth. This profile has been deduced from data described in New (1988) and Pingree \& New (1991). The dominant forcing frequency in the Bay of Biscay is the semidiurnal tide of frequency $\omega_{B B}=1.41 \times 10^{-4} \mathrm{rad} \mathrm{s}^{-1}$, corresponding to a period of $12.42 \mathrm{~h}$.

Let us see how the far-field condition applies to the configurations just described. In order to compute the phase speeds of the different internal wave modes, we introduce rotation in $(4.2 a)$,

$$
\frac{\mathrm{d}^{2} W}{\mathrm{~d} z^{2}}+K^{2} \frac{N^{2}(z)-\Omega^{2}}{\Omega^{2}-f_{45^{\circ}}^{2}} W(z)=0,
$$

where $f_{45^{\circ}}=10^{-4} \mathrm{rad} \mathrm{s}^{-1}$ is the Coriolis parameter at a latitude of $45^{\circ}$. The rest of the procedure is as in $\S 4$. 


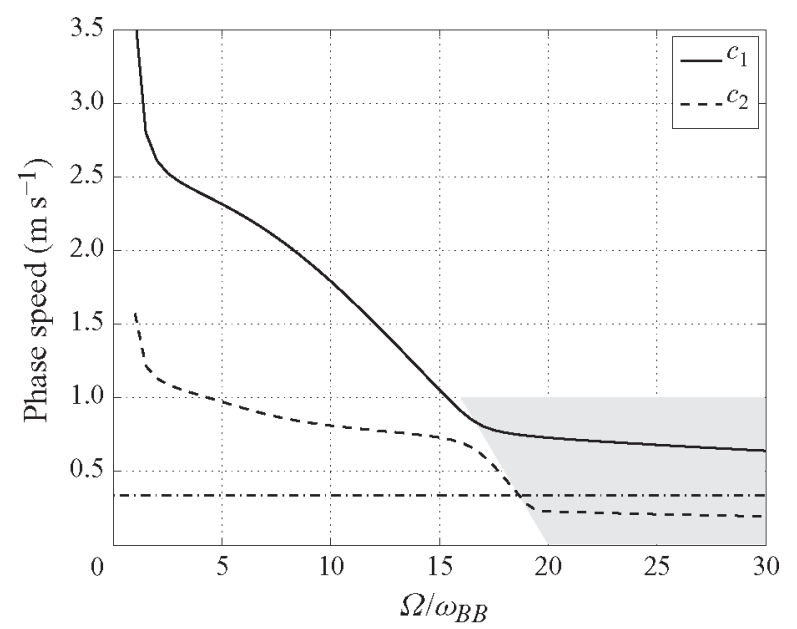

Figure 10. Phase speeds of the first two internal modes versus their frequency for the stratification displayed in figure 9 and with rotation. The grey zone approximately corresponds to the regime for which internal waves are trapped in the seasonal pycnocline. The dash-dotted line displays the horizontal phase speed of the beam in the Bay of Biscay, estimated from non-hydrostatic numerical simulations by Gerkema et al. (2004) and corresponding to a horizontal wavelength of the beam of $15 \mathrm{~km}$.

Before going into the details of the different regimes, let us set as a convention that a mode- $n$ wave of frequency $\Omega$ will be trapped in a given layer if (i) $\left(N^{2}-\Omega^{2}\right) /\left(\Omega^{2}-f_{45^{\circ}}^{2}\right)$ is positive throughout the layer and negative in the layers located immediately above and below it and (ii) if $W_{n}(z)$ (as defined in $(4.2 a)$ ) has its absolute maximum value in this layer. This trapped regime is shown in grey in figure 10. Note that for such high frequencies, rotation could be neglected in (6.1) but has an important role in setting the angle of the beam and therefore its horizontal wavelength. The typical phase speeds for the mode- 1 and mode- 2 trapped internal waves are approximately 0.7 and $0.2 \mathrm{~m} \mathrm{~s}^{-1}$, respectively. In order to match these phase speeds, an internal tidal beam of frequency $\omega_{B B}$ should have a dominant horizontal wavelength of 31 and $9 \mathrm{~km}$, respectively. Observations being scarce, the dominant horizontal wavelength of the beam in the Bay of Biscay just under the point of impact has to be estimated from previous numerical simulations. For example, figure 7 of Gerkema, Lam \& Maas (2004) shows a beam, whose horizontal wavelength is about $15 \mathrm{~km}$ (as computed from the horizontal dependency of the phase at a given vertical location). Regarding the far-field condition, $v_{\phi}$ is closer to $c_{2}$ than to $c_{1}$ in the trapped regime, the situation being generally intermediate. The discussion at the end of $\S 6.1$ shows that it is then possible that mode-1 ISWs are generated, coexisting with mode-2 ISWs, which are much harder to detect in the ocean.

We now move on to the near-field condition. Mode-1 ISWs are observed in the Bay of Biscay and the near-field condition only applies for modes strictly higher than 1 . As stated earlier in $\S 5, \mu_{2}$ should be noticeably higher than unity for mode-1 ISWs to be possibly generated. Let us examine whether this condition can be satisfied in the case of the Bay of Biscay. Assuming that the seasonal pycnocline is defined by $N(z)>3.5 \times 10^{-3} \mathrm{rad} \mathrm{s}^{-1}$, it is located between $z_{1}=-158 \mathrm{~m}$ and $z_{2}=-18 \mathrm{~m}$, meaning that the thickness of the pycnocline is $\delta_{p}=140 \mathrm{~m}$. As we are considering linear dynamics, the only frequency we are considering is $\omega_{B B}$ and the slope of the internal wave characteristics is then $\sqrt{\left(\omega_{B B}^{2}-f_{45^{\circ}}^{2}\right) /\left(N^{2}-\omega_{B B}^{2}\right)}$. Therefore, using the same 
notation as in $\S 5, \Delta x$ now has to verify the following condition:

$$
\Delta x=\int_{z_{1}}^{z_{2}} \mathrm{~d} z \sqrt{\frac{N^{2}(z)-\omega_{B B}^{2}}{\omega_{B B}^{2}-f_{45^{\circ}}^{2}}}=\delta_{p} \sqrt{\frac{N_{i}^{2}-\omega_{B B}^{2}}{\omega_{B B}^{2}-f_{45^{\circ}}^{2}}} .
$$

Then, $N_{i}$ is defined by

$$
N_{i}^{2}=\omega_{B B}^{2}+\frac{\omega_{B B}^{2}-f_{45^{\circ}}^{2}}{\delta_{p}^{2}}\left(\int_{z_{1}}^{z_{2}} \mathrm{~d} z \sqrt{\frac{N^{2}(z)-\omega_{B B}^{2}}{\omega_{B B}^{2}-f_{45^{\circ}}^{2}}}\right)^{2} .
$$

We find $N_{i}=8.4 \times 10^{-3} \mathrm{rad} \mathrm{s}^{-1}$. Once rotation is also included in the definition of $\theta_{i}$, the procedure is the same as in $\S 5$. In order to satisfy $\mu_{2}=1$, the wave beam should have a dominant horizontal wavelength of $24 \mathrm{~km}$, which is higher than the dominant horizontal wavelength of the beam in the Bay of Biscay as estimated earlier. Moreover, in order to satisfy $\mu_{3}=1$, the wave beam should have a horizontal wavelength of $12 \mathrm{~km}$. The near-field condition therefore predicts here that we are in an intermediate case between the generation of mode- 2 and mode- 3 ISWs. These considerations lead us to the conclusion that this simple model is quantitatively less suitable than the far-field approach when it comes to realistic conditions, one possible reason being the large uncertainties on the parameters that enter the model.

In comparison, the arguments on which the far-field approach is based are much more robust: for a given mode number $n$, trapped pycnocline waves with different frequencies propagate with phase speeds that are close to each other, which is therefore the approximate propagation speed of any mode- $n$ ISW trapped in the pycnocline. A beam (or any other disturbance) should therefore have a horizontal phase speed that is close to this value to efficiently force mode- $n$ ISWs (provided that the forcing is strong enough for nonlinear effects to develop within its direct range).

\section{Conclusion}

We addressed the local generation of ISWs, when an internal wave beam impinges on a pycnocline. Nonlinear, non-hydrostatic numerical experiments were conducted for this purpose, the vertical density profile being continuous and made of a lower layer with constant value $N_{0}$ of the Brunt-Väisälä frequency, a pycnocline of finite thickness and a thin homogeneous upper layer. This work complements previous theoretical (Gerkema 2001; Akylas et al. 2007) and experimental (Delisi \& Orlanski 1975) studies in which the thickness of the pycnocline was vanishingly small (i.e. an interface).

We showed that ISWs can be generated and that the finite thickness of the pycnocline, whose role is fundamental, allows different vertical modes to be excited, depending on the parameters. We ran numerical experiments showing that modes-1, -2 or -3 internal solitary waves can develop. We next proposed two different approaches to predict the conditions of occurrence of mode- $n$ internal solitary waves.

In the 'far-field' approach, that is, when harmonics of the forcing frequency have appeared, we showed that the observed mode- $n$ weakly nonlinear pycnocline waves have the same horizontal phase speed as the incident wave beam. We demonstrated this result heuristically by using a modal decomposition based upon the actual density profile and a frequency of value higher than $N_{0}$. This result allowed us to design numerical experiments so that a mode $-1,-2$ or -3 pycnocline wave develops, the 
density jump across the pycnocline increasing with the mode number (or equivalently, the horizontal wavelength of the wave beam decreasing with the mode number).

One might wonder why the phase speed of a beam should be matched with the phase speed of trapped waves, which are physically some distance away from the beam. We observed in $\S 3.2$ that the evolution towards ISWs actually takes one or two wavelengths only, a much shorter distance than for weakly nonlinear waves described e.g. by a KdV model. This is very likely due to the large amplitude of the forcing, which allows the development of nonlinear structures in its vicinity that propagate with the same speed as the forcing phase speed.

The 'near-field' approach consists of deriving simple geometrical conditions ensuring that a mode- $n$ wave develops at the beam impact (the model holds for $n \geqslant 2$ only). In this approach, the dynamics is linear, a simple plane wave is considered in place of a wave beam and a piecewise three-layer stratification is assumed. We showed that a mode- $n$ wave is forced if a simple relation is satisfied which involves the thickness of the pycnocline, the angle of the refracted internal wave in the pycnocline and the horizontal wavelength of this wave. In spite of its simplicity, this model gave a good quantitative agreement with our numerical data for $n \geqslant 2$. It explains why a beam with a short wavelength generates high-mode waves or, equivalently, why such high-mode waves are favoured by a strongly stratified pycnocline. When related to the far-field approach, this model provides a simple analytical expression for the phase speed of a mode- $n$ ISW.

We discussed the more general situation in which the wave beam has an intermediate phase speed between those of modes $n$ and $n+1$. We conjectured from an example that both modes could be selected in this case and that the lower the mode number is, the larger is its bandwidth. The computation of the amplitude of those modes is necessary to answer precisely this problem. This requires solving the nonlinear non-hydrostatic Boussinesq equations forced by the wave beam, a rather involved task.

The predictions of the two selection conditions derived in this paper were eventually examined in the more realistic context of the Bay of Biscay, in which mode-1 ISWs are observed. The far-field approach predicts a situation that is intermediate between the selection of mode- 1 and mode- 2 ISWs and an agreement with the observations is therefore possible (for more details, see Grisouard \& Staquet 2010). In contrast, the near-field approach does not seem to be quantitatively reliable when applied to this realistic configuration, as it predicts a situation that is intermediate between the selection of mode- 2 and mode- 3 ISWs. However, the application of the latter selection rule requires several simplifications, which leads to strong uncertainties in the parameters that enter the model.

Independent of the validity of these selection rules, it remains to investigate whether the mechanism leading to the generation of ISWs remains valid in a real ocean. The beams may have a three-dimensional structure and the generation process as a whole might be sensitive to perturbations from other structures such as eddies or other internal waves. The latter is the subject of a study by Grisouard \& Staquet (2010).

We thank the experimental team for having shared their early results, especially L. Gostiaux, M. Mercier and M. Mathur. M. Mathur is also acknowledged for his help during the revision of this paper. The detailed comments of the anonymous referees greatly helped to improve this article. N.G. is supported by a grant from the Direction Générale de l'Armement. This work has been supported by ANR contracts 
TOPOGI-3D (05-BLAN-0176) and PIWO (08-BLAN-0113). Numerical experiments were performed on the French supercomputer centre IDRIS, through contracts 070580 and 080580.

\section{REFERENCES}

Akylas, T. R., Grimshaw, R. H. J., Clarke, S. R. \& Tabaei, A. 2007 Reflecting tidal wave beams and local generation of solitary waves in the ocean thermocline. J. Fluid Mech. 593, 297313.

Azevedo, A., Da Silva, J. C. B. \& New, A. L. 2006 On the generation and propagation of internal solitary waves in the southern Bay of Biscay. Deep-Sea Res. I 53, 927-941.

Delisi, D. P. \& Orlanski, I. 1975 On the role of density jumps in the reflexion and breaking of internal gravity waves. $J$. Fluid Mech. 69, 445-464.

Gerkema, T. 2001 Internal and interfacial tides: Beam scattering and local generation of solitary waves. J. Mar. Res. 59, 227-255.

Gerkema, T., Lam, F.-P. A. \& MaAs, L. R. M. 2004 Internal tides in the Bay of Biscay: Conversion rates and seasonal effects. Deep-Sea Res. II 51, 2995-3008.

Gostiaux, L. \& Dauxois, T. 2007 Laboratory experiments on the generation of internal tidal beams over steep slopes. Phys. Fluids 19 (2), 028102.

Grisouard, N. \& Staquet, C. 2010 Numerical simulations of the local generation of internal solitary waves in the Bay of Biscay. Nonlinear Process. Geophys. 17, 575-584.

Helfrich, K. R. \& Melville, W. K. 2006 Long nonlinear internal waves. Annu. Rev. Fluid Mech. 38, 395-425.

Konyaev, K., Sabinin, K. \& Serebryany, A. 1995 Large-amplitude internal waves at the Mascarene Ridge in the Indian Ocean. Deep-Sea Res. I 42, 2075-2081.

LeBlond, P. H. \& Mysak, L. A. 1978 Waves in the Ocean. Elsevier.

Lighthill, J. 1978 Waves in Fluids. Cambridge University Press.

Marshall, J., Hill, C., Perelman, L. \& Adcroft, A. 1997 Hydrostatic, quasi-hydrostatic, and nonhydrostatic ocean modeling. J. Geophys. Res. 102, 5733-5752.

Mathur, M. \& PeAcock, T. 2009 Internal wave beam propagation in non-uniform stratifications. J. Fluid Mech. 639 (1), 133-152.

Maugé, R. \& Gerkema, T. 2008 Generation of weakly nonlinear nonhydrostatic internal tides over large topography: a multi-modal approach. Nonlinear Process. Geophys. 15, 233244.

New, A. L. 1988 Internal tidal mixing in the Bay of Biscay. Deep-Sea Res. 35 (5), 691-709.

New, A. L. \& Pingree, R. D. 1990 Large-amplitude internal soliton packets in the central Bay of Biscay. Deep-Sea Res. 37 (3), 513-524.

New, A. L. \& Pingree, R. D. 1992 Local generation of internal soliton packets in the central Bay of Biscay. Deep-Sea Res. 39 (9), 1521-1534.

New, A. L. \& DA Silva, J. C. B. 2002 Remote-sensing evidence for the local generation of internal soliton packets in the central Bay of Biscay. Deep-Sea Res. I 49, 915-934.

Ostrovsky, L. A. \& Stepanyants, Y. A. 1989 Do internal solitons exist in the ocean? Rev. Geophys. 27, 293-310.

Pingree, R. D. \& New, A. L. 1991 Abyssal penetration and bottom reflection of internal tidal energy in the Bay of Biscay. J. Phys. Oceanogr. 21, 28-39.

DA Silva, J. C. B., New, A. L. \& Azevedo, A. 2007 On the role of SAR for observing 'local generation' of internal solitary waves off the Iberian Peninsula. Can. J. Remote Sens. 33 (5), 388-403.

Da Silva, J. C. B., New, A. L. M. \& Magalhaes, J. M. 2009 Internal solitary waves in the Mozambique Channel: Observations and interpretation. J. Geophys. Res. 114, C05001.

Staquet, C., Sommeria, J., Goswami, K. \& Mehdizadeh, M. 2006 Propagation of the Internal Tide from a Continental Shelf: Laboratory and Numerical Experiments. In Sixth International Symposium on Stratified Flows, Perth, Australia, pp. 539-544.

Thomas, N. H. \& Stevenson, T. N. 1972 A similarity solution for viscous internal waves. J. Fluid Mech. 54, 495-506. 
THorpe, S. A. 1987 Reflection of internal waves from a uniform slope. J. Fluid Mech. 178, 279-302. THorpe, S. A. 1998 Nonlinear reflection of internal waves at a density discontinuity at the base of the mixed layer. J. Phys. Oceanogr. 28, 1853-1860.

Zhang, H. P., KING, B. \& SwINney, H. L. 2007 Experimental study of internal gravity waves generated by supercritical topography. Phys. Fluids 19 (9), 096602.

Zhang, K. Q. \& Marotzke, J. 1999 The importance of open-boundary estimation for an Indian Ocean GCM-data synthesis. J. Mar. Res. 57 (2), 305-334. 